\title{
Análisis del confort y el comportamiento higrotérmico de sistemas constructivos tradicionales y actuales en viviendas de Santa Ana-Ciudad Colón (Costa Rica)
}

\author{
Analysis of thermal and humidity comfort of traditional versus current \\ construction systems in Santa Ana-Ciudad Colón (Costa Rica) \\ B. G. Rodrigo $^{(*)}$, J. C. Sanabria ${ }^{(* *)}$, M. Marchamalo ${ }^{(*)}$, M. Umaña $a^{(* *)}$
}

\section{RESUMEN}

El objetivo de la investigación es comparar el confort de viviendas tradicionales de Santa Ana-Ciudad Colón (Costa Rica), construidas en adobe con cubierta cerámica, frente a los sistemas constructivos actualmente empleados. Para analizar el confort se diseñó un experimento de medición real de parámetros climáticos en una muestra de viviendas de distintos sistemas constructivos. Los datos se interpretaron según la norma ISO 7730 y la norma 55 de ASHRAE considerando las variables de Voto Medio Estimado (PMV), Porcentaje de Personas Insatisfechas (PPD) y la sensación térmica del cuerpo para distintos escenarios de humedad, temperatura, movimiento del aire, actividad física y abrigo del usuario.

Los resultados muestran que, en este clima, el confort térmico aumenta en viviendas con muros exteriores pesados y cubiertas aisladas que eviten la captación excesiva de calor. Las viviendas tradicionales presentan más del $97 \%$ de los datos dentro de los intervalos establecidos de ambiente confortable.

$113-113$

Palabras clave: Confort térmico, clima tropical, adobe, hormigón, sistemas constructivos.

\section{SUMMARY}

This research was carried out in Santa AnaCiudad Colón (Costa Rica). The aim of this study is to compare comfort between traditional (adobe houses with ceramic tile roofs) and modern (block or precast concrete houses with galvanized sheet roofs) building systems. To analyze comfort it was designed an experiment measuring real climatic parameters in a set of houses of different building systems. Data was analysed using ISO 7730 and ASHRAE Standard 55 rules, considering variables such as the predicted mean vote (PMV), predicted percentage dissatisfied (PPD) and the thermal sensation of the body for different scenarios of humidity, temperature, wind, user activity and shelter.

Results show that comfort increases when the house has thick exterior walls under studied climate conditions that ensure thermal inertia and insulated roof covers to avoid over-abstraction of heat. Traditional homes present comfortable conditions in at least $97 \%$ of the recorded time while current houses present comfortable conditions
Keywords: Thermal comfort, tropical climate, adobe, concrete, construction systems.

(*) Universidad Politécnica de Madrid. Madrid (España)

(**) Universidad Veritas. San José (Costa Rica)

$(* * *)$ Arquitecto. San José (Costa Rica)

Persona de contacto/Corresponding author: beatriz.gonzalez.rodrigo@upm.es (B. G. Rodrigo)

Fecha de recepción: 09-06-10 Fecha de aceptación: 24-03-11 


\section{INTRODUCCIÓN}

Uno de los principales objetivos de las construcciones a lo largo de la historia es proporcionar las condiciones óptimas de confort térmico para la especie humana. Desde los inicios de la civilización, el ser humano ha modificado su hábitat para cobijarse del clima y de otros agentes externos. En cambio, gran parte de los edificios modernos ponen gran énfasis en el diseño, los materiales y la construcción, pero se olvidan de que su principal objetivo es lograr un ambiente interior confortable (1).

La sensación térmica experimentada por un ser humano está relacionada, principalmente, con el equilibrio térmico global del cuerpo. Tal equilibrio depende de la actividad física y de la vestimenta del sujeto así como de los parámetros ambientales: temperatura del aire, temperatura radiante media, velocidad del aire y humedad del aire (2) (3). Por lo tanto, el confort se puede definir como la buena sensación física y mental de los moradores de una edificación (4). El técnico debe buscar con sus diseños obtener soluciones que satisfagan las necesidades psicológicas, sociales y funcionales, así como las físicas y fisiológicas, de los ocupantes en el marco de unas circunstancias topográficas, climáticas y económicas dadas.

El confort térmico puede medirse a través de dos variables: el voto medio estimado (PMV-predicted mean vote) y el porcentaje de personas insatisfechas (PPD-predicted percentage dissatisfied) (2) (3). La primera variable, PMV, refleja el valor de los juicios o votos emitidos por un grupo numeroso de personas respecto de una escala de sensación térmica de -3 a 3, es decir siete niveles (frío, fresco, ligeramente fresco, neutro, ligeramente calurosos, caluroso y muy caluroso). Esta variable PMV está basada en el equilibrio térmico del cuerpo humano, considerando que la producción interna de calor del cuerpo se equilibra con su pérdida hacia el ambiente. La variable PPD permite determinar el porcentaje de personas que sienten mucho frío o calor, es decir, que la sensación provocada por el entorno les resulte desagradable.

Las estrategias de diseño para la obtención de un máximo confort térmico en una zona determinada deben responder a las condiciones climáticas locales, por lo tanto, construcciones tradicionales similares son empleadas en diferentes partes del mundo con clima similar. Las tipologías constructivas vernáculas se encuentran definidas más por las zonas climáticas que por las fronteras administrativas tradicionales (5).
En el clima tropical húmedo, como el analizado en Costa Rica, los vientos son generalmente suaves, variables en velocidad, pero casi constantes en dirección. La ventilación es la principal estrategia a considerar para conseguir condiciones de bienestar en este clima húmedo (6). El efecto del movimiento de aire repercute en el enfriamiento directo del aire interno cuando el exterior es más frío que el interior y al aumento de confort térmico por medio en la sensación de frescor (7). Esta sensación es debida a la pérdida de calor por convección y al aumento de evaporación del cuerpo. Por lo tanto, con objeto de alcanzar el confort físico es necesario conseguir la disipación térmica del cuerpo hacia el medio o evitar la captación y acumulación del calor. El confort mejora haciendo que la brisa exterior circule, no solamente a través del edificio, sino también sobre las superficies corporales de los ocupantes (8).

El clima de la región analizada presenta temperaturas del aire moderadamente elevadas con variaciones pequeñas entre el día y la noche. El vapor de agua y las nubes espesas actúan como filtros ante la radiación directa del sol, por lo que ésta se reduce y difunde. A pesar de que la radiación solar queda atenuada por las nubes, es una importante fuente de calor, por lo que su captación por el edificio debe evitarse. La situación geográfica de Costa Rica, a $10^{\circ}$ de latitud norte, determina que los rayos solares incidan muy verticalmente sobre la superficie, por lo tanto en este clima la cubierta es un elemento esencial, con mayor importancia que los muros (9). Este elemento puede estar sometido a grandes fluctuaciones de temperatura a lo largo del día dependiendo del tipo de cubierta y del color (4). Las cubiertas deben jugar un doble papel: 1) proporcionar sombra sobre las superficies verticales, ya sean paredes opacas o aberturas y 2) evitar un exceso de captación de calor y transmisión dentro del edificio.

Debido a la gran variación climática que presenta Costa Rica a lo largo de su territorio, no existe un tipo único de construcción tradicional, no obstante, la mayoría de las viviendas tradicionales eran concebidas con una ventilación cruzada para asegurar el movimiento del aire dentro del edificio. Esta ventilación en muchos casos se sacrificaba cuando iba creciendo la familia y la vivienda iba aumentando. En la zona de estudio, se han localizado viviendas tradicionales construidas en adobe con cubiertas resueltas con una teja cerámica apoyada sobre caña brava y todo ello sostenido por pares o correas de madera. Actualmente, un porcentaje muy alto de las viviendas costarricenses, independientemente de las 
características climáticas de la zona, están construidas con bloques de hormigón o elementos prefabricados de hormigón (en una sola hoja) y la cubierta está resuelta con un cerramiento de chapa metálica galvanizada colocada sobre perfilería metálica, sin aislamiento o cámara de aire debajo de la misma, ni ventilación cruzada.

El presente estudio tiene por objetivo realizar una evaluación experimental comparativa entre el confort térmico en la localidad de Santa Ana (Costa Rica) del sistema constructivo tradicional en adobe frente a los empleados actualmente de bloque de hormigón y el sistema prefabricado de montantes y paneles de hormigón. La hipótesis de partida es que la edificación actual en este país no presenta mejor confort que las viviendas tradicionales.

\section{METODOLOGÍA}

\subsection{Zona de estudio}

La investigación fue realizada en Santa AnaCiudad Colón, localidad próxima a la capital, San José. Santa Ana está ubicada en la zona central a 900 metros sobre el nivel del mar, con una temperatura media de $23,7^{\circ} \mathrm{C}$ que varía anualmente una media de $3{ }^{\circ} \mathrm{C}$ en promedio (10). Las temperaturas disminuyen en la época húmeda (Junio-Noviembre) debido a que las nubes reflejan parte de la de temperaturas es de $9,5{ }^{\circ} \mathrm{C}$ (10), siendo mayor en la estación seca que en la lluviosa.

Las principales influencias eólicas de Costa Rica son: a) sistema de vientos globales alisios de componente NE, b) vientos orográficos y c) las brisas tierra-mar. En la zona de estudio influye principalmente el sistema de vientos globales. Costa Rica recibe entre 1.500 y $6.000 \mathrm{~mm}$ anuales de lluvia según las zonas. En la zona de Ciudad Colón-Santa Ana la lluvia media es de 2.137 mm (10).

\subsection{Diseño experimental}

Para el estudio se seleccionaron casas pequeñas, de menos de $120 \mathrm{~m}^{2}$, aisladas, radiación del sol. La variación diurna media

en situaciones similares de obstáculos o edificaciones en las inmediaciones y con una topografía y entorno parecido. Se seleccionaron dos casas con sistema constructivo tradicional de la zona: muro de adobe y cubierta de teja cerámica sobre vigas de madera. Igualmente se escogieron dos viviendas fabricadas empleando los sistemas constructivos más generalizados en el país actualmente: bloques de hormigón y sistemas con montantes y paneles prefabricados de hormigón. En los dos últimos casos, las viviendas presentaban cerramiento de cubierta de chapa metálica zincada con un falso techo. Las dos primeras viviendas nombradas son de autoría anónima, aunque una de ellas ha sido objeto de una remodelación mediante un proyecto de un técnico, mientras que las otras dos han sido diseñadas por técnicos.

Se considera que la influencia, en el presente estudio, de los comportamientos o hábitos del usuario de las viviendas es baja en comparación con el tipo de materiales empleados en muros y cubierta. En la cultura costarricense, las casas aisladas y en el campo suelen permanecer abiertas siempre que estén habitadas y las viviendas suelen tener aberturas permanentes que aseguran una ventilación constante. Esta ventilación permanente era especialmente importante en la vivienda 1, la única no habitada de las cuatro analizadas. De hecho se realizó un estudio estadístico obteniendo que no había diferencias significativas entre los datos obtenidos a lo largo de los días de la semana en las viviendas 2,3 y 4 .

La toma de datos de las viviendas se realizó durante el mes de junio (bloques de hormigón y adobe 2), julio (adobe 1) y agosto (sistema prefabricado de hormigón). El histórico de la media de las temperaturas y humedades máximas y mínimas mensuales en la zona de estudio queda reflejado en la Tabla 1, presentando, durante estos meses la humedad relativa máxima de $97 \%$ y mínima de $53 \%$.

Tabla 1

Histórico de temperatura y precipitación mensuales medias en la zona (Datos obtenidos del histórico (1957-2002) del Dr. L, Fournier en Ciudad Colón) y de humedad relativa del aire mensuales medias (Datos obtenidos del Instituto Meteorológico Nacional)

\begin{tabular}{|c|c|c|c|c|c|c|c|c|c|c|c|c|c|}
\hline & & Ene. & Feb. & Mar. & Abr. & May. & Jun. & Jul. & Ago. & Sep. & Oct. & Nov. & Dic. \\
\hline \multirow{2}{*}{$\begin{array}{l}\text { Temperatura } \\
\text { del aire }{ }^{\circ} \mathrm{C}\end{array}$} & Máx. mensuales & 28,0 & 29,6 & 31,1 & 31,1 & 29,1 & 27,8 & 28,0 & 27,7 & 26,8 & 26,1 & 26,0 & 26,8 \\
\hline & Mín. mensuales & 18,8 & 19,1 & 18,9 & 19,9 & 20,1 & 19,4 & 19,5 & 19,2 & 18,9 & 18,8 & 19,1 & 19,3 \\
\hline \multirow{2}{*}{$\begin{array}{l}\text { Humedad } \\
\text { relativa }(\%)\end{array}$} & Máx. mensuales & 65 & 65 & 65 & 75 & 75,4 & 80 & 80 & 80 & 85 & 85 & 70 & 66 \\
\hline & Mín. mensuales & 40 & 40 & 40 & 43,2 & 46,7 & 55 & 55 & 55 & 60 & 60 & 64 & 61 \\
\hline \multicolumn{2}{|c|}{ Pluviosidad $(\mathrm{mm})$} & 6,7 & 22,5 & 39,8 & 102,7 & 278,1 & 296,3 & 162,8 & 252,0 & 409,2 & 394,1 & 145,0 & 33,7 \\
\hline
\end{tabular}


1. Vistas de la casa tradicional de adobe (vivienda 1).

2. Vistas de la casa tradicional de adobe (vivienda 2).
- Viviendas con sistema constructivo tradicional (Viviendas 1 y 2 ):

La vivienda de adobe 1 (a partir de ahora vivienda 1) tiene las siguientes características:

- Está orientada Norte-Sur con aberturas principales menores al $40 \%$ de vidrio fijo, ventanas abatibles y de guillotina de pequeño tamaño.

- Casa aislada, sin colindancia.

- Pavimento cerámico, madera y piedra.

- Cubierta de teja cerámica colocada a 4 aguas con falso techo en madera y caña brava.

- Ventilación directa.

- lluminación directa por medio de ventanas y entrada de luz cenital (usada en varios espacios).

- La casa no está habitada.

La vivienda de adobe 2 (a partir de ahora vivienda 2) tiene las siguientes características:

- Está orientada Norte-Sur con una nueva ampliación Este-Oeste con aberturas principales menores al $40 \%$.

- Casa aislada, sin colindancia.

- Pavimento cerámico y piedra (terraza).

- Cubierta teja cerámica, con chapa metálica y con falso techo de caña brava.

- Vidrio fijo, ventanas abatibles, de guillotina y de celosías (en general de pequeño tamaño).

- Ventilación directa.

- Iluminación directa por medio de ventanas y entrada de luz cenital (usada en varios espacios).
- Ubicada en terreno plano amplio y con mucha vegetación, el diseño es típico de las casas de adobe (el área original).

- La vivienda está habitada.

- Viviendas de sistemas constructivos actuales (Viviendas 3 y 4 ):

La vivienda aislada de bloques de hormigón (a partir de ahora vivienda 3) presenta las siguientes características:

- Está orientada de este a oeste con las principales aberturas al oeste y al sur mayores al $40 \%$ a través de vidrio fijo y ventanas abatibles.

- Casa aislada, sin colindancia.

- Construcción de hormigón con detalles en madera.

- Pavimento de hormigón.

- Cubierta con cerramiento metálico colocada a dos aguas con falso techo en madera.

- Ventilación directa y cruzada.

- Iluminación directa y abundante.

- La vivienda está habitada.

La vivienda aislada de sistema prefabricado de montantes y paneles de hormigón (a partir de ahora vivienda 4) presenta las siguientes características:

- Casa cuadrada con aperturas principales al norte y al oeste, menores al $40 \%$ a través de vidrio fijo y ventanas abatibles.

- Casa aislada, con una pequeña colindancia hacia el este.

- Pavimento de terrazo.
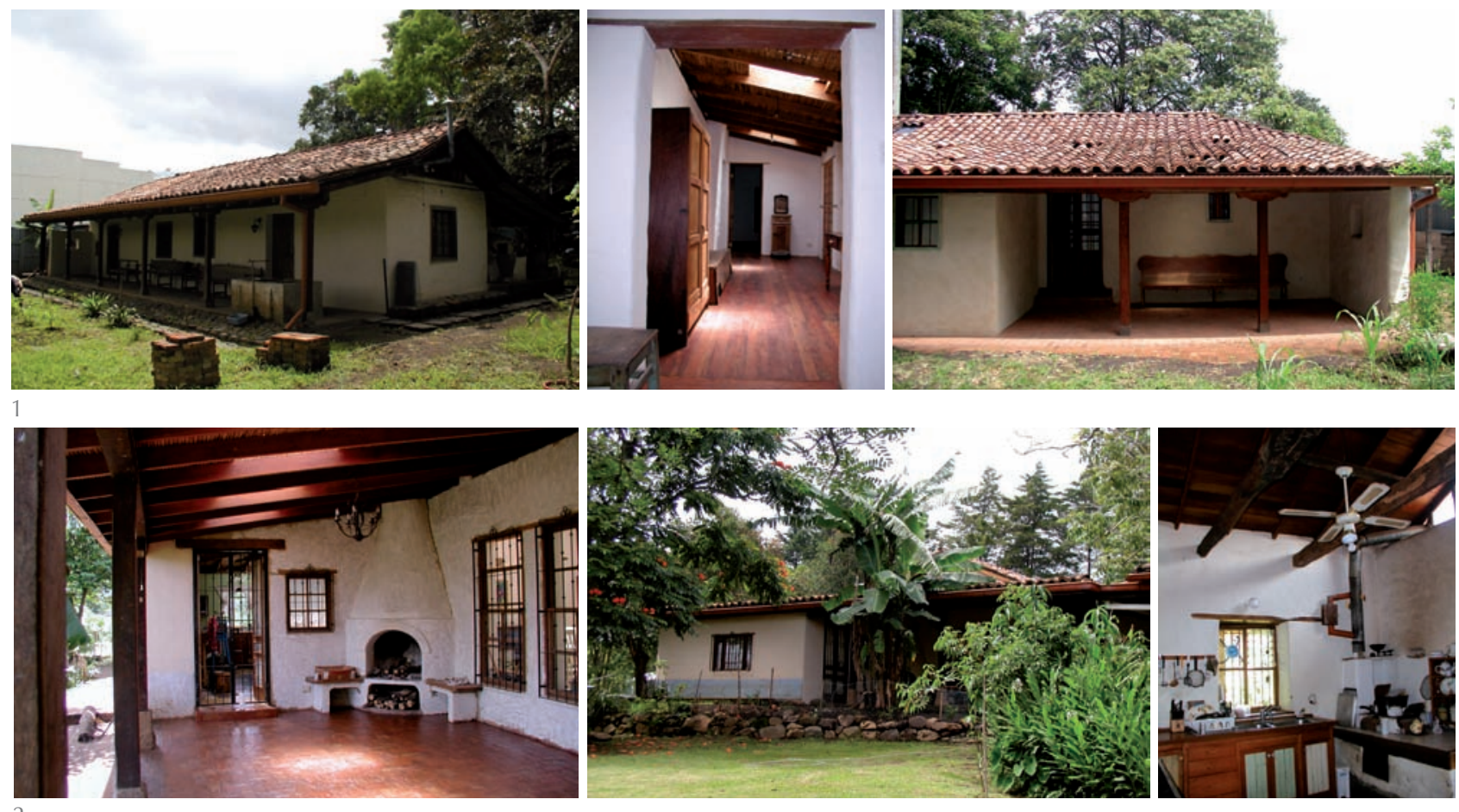

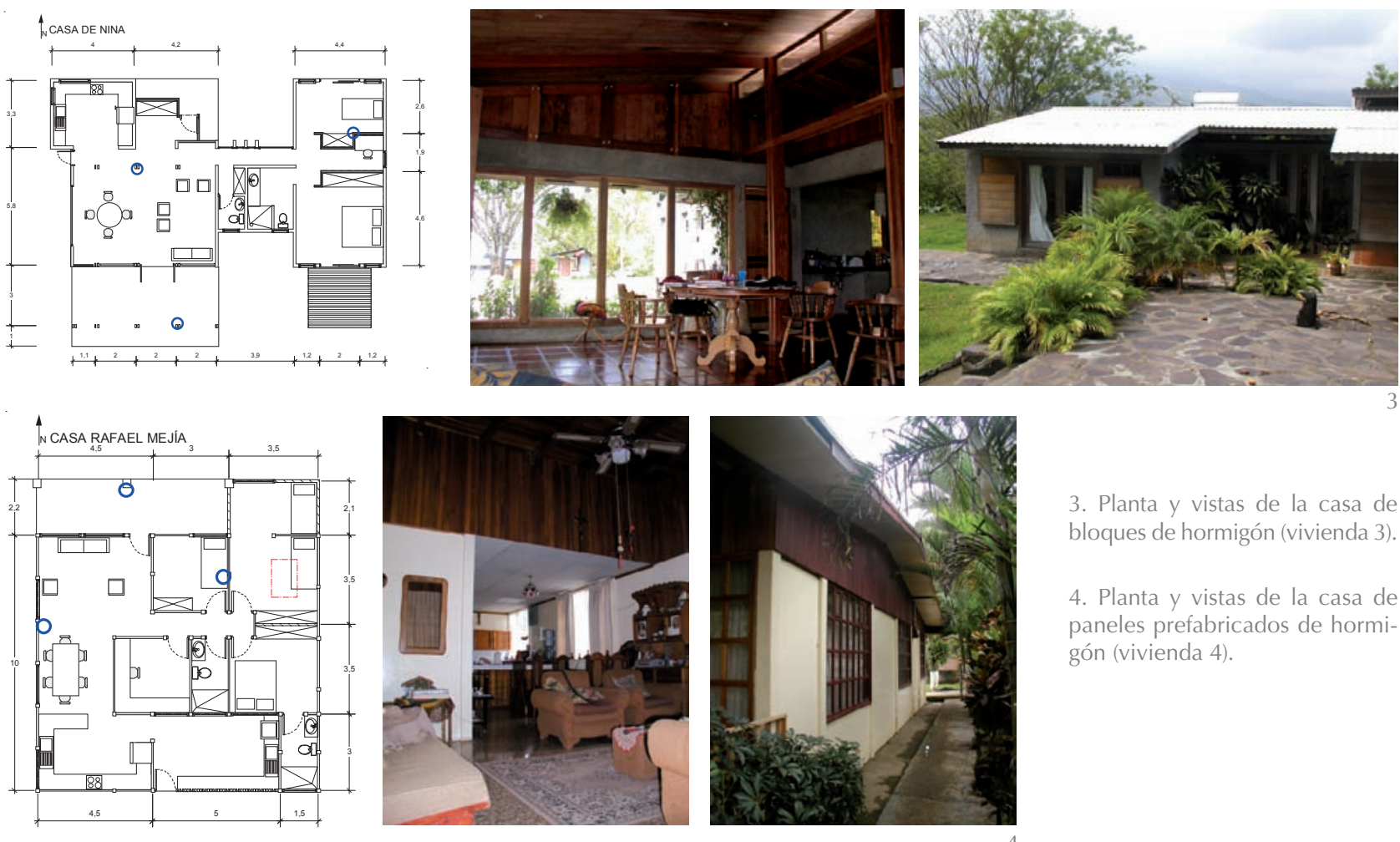

3. Planta y vistas de la casa de bloques de hormigón (vivienda 3).

4. Planta y vistas de la casa de paneles prefabricados de hormigón (vivienda 4).

- Cubierta con cerramiento metálico colocada a dos aguas con falso techo en madera en sala y placa de fibrocemento en habitaciones.

- Ventilación directa. La ventilación cruzada fue sacrificada por una ampliación de la vivienda.

- Iluminación escasa.

- La vivienda está habitada.

\subsection{Instrumentación y toma de datos}

Para la toma de datos se utilizaron tres termo-higrómetros HOBO RH Temp. Osent H08-004-02. Para el monitoreo se colocaron en los tres espacios que se consideraron importantes: el salón, el dormitorio y la terraza. Los aparatos fueron colocados en un intervalo de alturas de 1,8 y 2,2 metros. El termo-higrómetro de la terraza o corredor estaba colocado al abrigo de la luz directa de forma que los datos obtenidos no estuvieran influidos por el asoleamiento. Los termo-higrómetros registraron mediciones cada 10 minutos de temperatura y humedad durante un período igual o superior a 7 días que estuvieron colocados. La toma de datos se realizó en todas las viviendas en la época húmeda (de junio a octubre).

\subsection{Análisis de datos}

a) Tablas de Mahoney

Para el análisis de los datos se consideró el diseño ideal del edificio según las tablas de Mahoney (8) para el clima de Santa Ana-Ciudad Colón. Estas tablas fueron desarrolladas para ayudar en el diseño de las viviendas en los países tropicales a partir de los siguientes datos: longitud, latitud y altitud de la zona, media, máxima y mínima mensual de las temperaturas, humedad relativa mensual, precipitaciones medias mensuales y dirección del viento predominante mensualmente. A partir de los datos climáticos de esta zona (10) y empleando las tablas de Mahoney se obtiene que durante el mes de mayo la ventilación es esencial, siendo recomendable durante los meses de septiembre y octubre. La protección de la lluvia es fundamental durante la estación lluviosa mientras que en la estación seca, especialmente en los meses de febrero, marzo y abril se requiere amortiguar las altas temperaturas en las horas centrales del día.

A continuación se enumeran los aspectos más significativos que debe cumplir una vivienda en estas condiciones climáticas, según lo obtenido del análisis realizado con las Tablas de Mahoney:

- Los edificios deben estar orientados al Norte y al Sur (a lo largo del eje Este-Oeste).

- Los edificios deben tener una orientación simple con dispositivos que permitan la circulación del aire.

- Las aberturas deben ser medianas, entre el 20 y $40 \%$, evitando la luz directa y asegurando estar protegidas de la lluvia.

- Las paredes externas deben ser pesadas.

- El tejado debe ser ligero pero bien aislado.

- Se necesitan medidas de protección contra las lluvias frecuentes, tal como galerías profundas, salientes amplios y corredores cubiertos. 
1 Versión 1.07 desarrollada por Charloie Huizenga y Marc Fountain.

\section{b) Intervalos de Confort}

Para determinar los intervalos de confort y las medias de las temperaturas se discriminó entre día y noche. Se consideró noche (entre las 22:00 y las 6:00h) aquellas horas en las que los habitantes de la vivienda están acostados y por lo tanto con una actividad muy baja y día las horas en las cuales los habitantes de la vivienda presentan una actividad. Por lo tanto, a la hora de determinar los intervalos de confort se tuvo en cuenta tanto esta diferencia de actividad como la variación de abrigo.

Para determinar los intervalos de confort se utilizó el programa UC Berkeley Thermal Confort Program ${ }^{1}$. Este programa se basa en las normativas ANSI- ASHRAE STD 5 (11) e ISO 7730 (3). Esta última determina que el grado de confort se obtiene para valores de PMV entre $-0,5$ y 0,5. No obstante, para este clima se puede considerar que inmediatamente por encima y por debajo de estos valores, existen unos intervalos en los que la sensación térmica del cuerpo sigue siendo de agradable, y por lo tanto se obtienen rangos aceptables de temperaturas. En base a esto, se han determinado cinco categorías de confort. Las categorías de confort consideradas son las siguientes: a) PMV entre $-0,5$ y 0,5 (según estándar ISO 7730); b) PMV mayor a 0,5 pero con una sensación térmica por debajo de $27^{\circ} \mathrm{C}$ (calor confortable) (11); c) PMV menor a $-0,5$ pero con una sensación térmica por encima del $18,6{ }^{\circ} \mathrm{C}$ (frío confortable) (11); d) y e) No existe confort debido a un exceso de frío o de calor.

Para la obtención de los intervalos de confort se ha tenido en cuenta las siguientes variables en la habitación en donde se realice el análisis: a) Temperatura y humedad relativa de la habitación (obtenidas a partir de los termo-higrómetros).

b) Temperatura radiante media. Variable que se obtiene a partir de la temperatura ambiente, la temperatura media global y la velocidad del aire.

c) Velocidad del aire. Se ha tomado un valor medio de $0,1 \mathrm{~m} / \mathrm{s}$. Se considera que la velocidad del aire no depende del comportamiento o los hábitos del usuario debido a que en esta zona las viviendas suelen tener zonas de ventilación permanente.

d) Actividad del metabolismo Se ha considerado que una persona durante el día tiene un metabolismo de 1,2 met, equivalente a la actividad sedentaria de estar leyendo o escribiendo en el ordenador. Durante la noche, su actividad metabólica es de 0,8 met, equivalente a estar tumbado (11). Se considera que 1,0 met es 58,2 watts $/ \mathrm{m}^{2}$.

e) Ropa que se lleva. Se considera que durante el día, el ocupante lleva la ropa de verano $(0,5$ clo (11)) y durante la noche, la ropa que lleva encima es equivalente a una manta (1,0 clo).

f) Datos fisiológicos: a) Temperatura inicial del cuerpo $36,5^{\circ}$; b) Masa corporal 70 $\mathrm{kg}$; c) Superficie corporal: 1,83 $\mathrm{m}^{2}$; tiempo de exposición a la temperatura: 60 min. Vasodilatación $120 \mathrm{l} / \mathrm{m}^{2} \mathrm{hr}-\mathrm{K}$, coeficiente de vasoconstricción 0,5 .

La Tabla 2 representa los intervalos de confort basados en la Norma ISO 7730 (3) y en ASHRAE Standard 55 (11) para los diferentes intervalos de humedad considerando las variables de actividad metabólica y cantidad de ropa descrita anteriormente.

Tabla 2

Intervalos de temperatura $\left({ }^{\circ} \mathrm{C}\right)$ para la obtención del confort según la norma ISO 7730 y la ASHRAE Std 55 para diferentes intervalos de humedad. Santa Ana, Costa Rica

\begin{tabular}{|c|c|c|c|c|c|c|}
\hline & Humedad & $\begin{array}{l}\text { No confort } \\
\text { por frío }\end{array}$ & $\begin{array}{l}\text { Confort por } \\
\text { debajo ISO }\end{array}$ & $\begin{array}{c}\text { Confort según } \\
\text { ISO }^{3}\end{array}$ & $\begin{array}{l}\text { Confort por } \\
\text { encima ISO } \text { ISO }^{4}\end{array}$ & $\begin{array}{l}\text { No confort } \\
\text { por calor }^{1}\end{array}$ \\
\hline \multirow{6}{*}{ 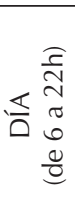 } & $30-40 \%$ & \begin{tabular}{l|l}
$18,7^{\circ} \mathrm{C}$ \\
\end{tabular} & \begin{tabular}{|l|l|} 
& $23,4^{\circ} \mathrm{C}$ \\
\end{tabular} & \begin{tabular}{|l|l|l} 
& $26,7^{\circ} \mathrm{C}$
\end{tabular} & \begin{tabular}{|l|l|l} 
& $28,1^{\circ} \mathrm{C}$
\end{tabular} & \\
\hline & $40-50 \%$ & $18,5^{\circ} \mathrm{C}$ & $23,2^{\circ} \mathrm{C}$ & $26,5^{\circ} \mathrm{C}$ & $27,7^{\circ} \mathrm{C}$ & \\
\hline & $50-60 \%$ & $18,4^{\circ} \mathrm{C}$ & $23,0^{\circ} \mathrm{C}$ & $26,2^{\circ} \mathrm{C}$ & $27,3^{\circ} \mathrm{C}$ & \\
\hline & $60-70 \%$ & $18,2^{\circ} \mathrm{C}$ & $22,8^{\circ} \mathrm{C}$ & $26,0^{\circ} \mathrm{C}$ & $26,9^{\circ} \mathrm{C}$ & \\
\hline & $70-80 \%$ & $18,1^{\circ} \mathrm{C}$ & $22,6^{\circ} \mathrm{C}$ & $25,7^{\circ} \mathrm{C}$ & $26,5^{\circ} \mathrm{C}$ & \\
\hline & $80-90 \%$ & $17,9^{\circ} \mathrm{C}$ & $22,4^{\circ} \mathrm{C}$ & $25,5^{\circ} \mathrm{C}$ & $26,1^{\circ} \mathrm{C}$ & \\
\hline \multirow{6}{*}{ 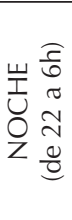 } & $30-40 \%$ & $19,5^{\circ} \mathrm{C}$ & $25,2^{\circ} \mathrm{C}$ & $28,0^{\circ} \mathrm{C}$ & $28,4^{\circ} \mathrm{C}$ & \\
\hline & $40-50 \%$ & $19,3^{\circ} \mathrm{C}$ & $25,0^{\circ} \mathrm{C}$ & $27,7^{\circ} \mathrm{C}$ & $28,0^{\circ} \mathrm{C}$ & \\
\hline & $50-60 \%$ & $19,2^{\circ} \mathrm{C}$ & $24,7^{\circ} \mathrm{C}$ & $27,4^{\circ} \mathrm{C}$ & $27,6^{\circ} \mathrm{C}$ & \\
\hline & $60-70 \%$ & $19,1^{\circ} \mathrm{C}$ & $24,4^{\circ} \mathrm{C}$ & $27,0^{\circ} \mathrm{C}$ & $27,3^{\circ} \mathrm{C}$ & \\
\hline & $70-80 \%$ & $18,9^{\circ} \mathrm{C}$ & $24,1^{\circ} \mathrm{C}$ & $26,7^{\circ} \mathrm{C}$ & $27,0^{\circ} \mathrm{C}$ & \\
\hline & $80-90 \%$ & $18,8^{\circ} \mathrm{C}$ & $23,8^{\circ} \mathrm{C}$ & $26,4^{\circ} \mathrm{C}$ & $26,7^{\circ} \mathrm{C}$ & \\
\hline
\end{tabular}

1 No existe confort debido a un exceso de frío o de calor

2 PMV menor a -0.5 pero con una sensación térmica por encima del $18,6^{\circ} \mathrm{C}$ (frío confortable según ASHAE Standard 55)

3 PMV entre - 0.5 y 0.5 (según estándar ISO 7730)

4 PMV mayor a 0.5 pero con una sensación térmica por debajo de $27^{\circ} \mathrm{C}$ (Calor confortable ASHAE Standard 55) 
Tabla 3

Medias y desviaciones típicas de las temperaturas y humedad obtenidas durante los días de toma de datos en las diferentes viviendas

\begin{tabular}{|c|c|c|c|c|c|c|c|c|c|}
\hline & & \multicolumn{4}{|c|}{ Temperatura ${ }^{\circ} \mathrm{C}$} & \multicolumn{4}{|c|}{ Humedad relativa $\%$} \\
\hline & & \multicolumn{2}{|c|}{ Día } & \multicolumn{2}{|c|}{ Noche } & \multicolumn{2}{|c|}{ Día } & \multicolumn{2}{|c|}{ Noche } \\
\hline & & Media & Desv & Media & Desv & Media & Desv & Media & Desv \\
\hline \multirow{3}{*}{$\begin{array}{l}\text { Sistema tradicional } \\
\text { (adobe 1) }\end{array}$} & salón & 21,67 & 0,88 & 20,25 & 0,64 & 74,22 & 2,43 & 75,69 & 1,05 \\
\hline & dormitorio & 22,09 & 0,62 & 21,32 & 0,45 & 72,05 & 1,47 & 72,22 & 0,78 \\
\hline & terraza & 21,83 & 1,53 & 19,62 & 0,50 & 73,47 & 5,53 & 78,05 & 2,02 \\
\hline \multirow{3}{*}{$\begin{array}{l}\text { Sistema tradicional } \\
\text { (adobe 2) }\end{array}$} & salón & 22,72 & 1,07 & 21,85 & 0,73 & 68,74 & 2,58 & 68,85 & 2,38 \\
\hline & habitación & 22,95 & 1,05 & 21,53 & 0,76 & 67,01 & 2,77 & 69,06 & 2,28 \\
\hline & terraza & 22,94 & 1,69 & 20,46 & 0,70 & 67,32 & 4,75 & 72,30 & 3,49 \\
\hline \multirow{3}{*}{$\begin{array}{l}\text { Sistema actual (fábrica } \\
\text { de hormigón) }\end{array}$} & salón & 26,90 & 2,02 & 24,29 & 0,83 & 58,49 & 8,44 & 64,03 & 5,04 \\
\hline & habitación & 27,48 & 2,22 & 24,38 & 1,05 & 55,93 & 7,65 & 61,66 & 5,10 \\
\hline & terraza & 25,74 & 3,07 & 22,44 & 1,48 & 62,20 & 12,81 & 71,01 & 8,38 \\
\hline \multirow{3}{*}{$\begin{array}{l}\text { Sistema actual (prefa- } \\
\text { bricado de hormigón) }\end{array}$} & salón & 25,47 & 2,18 & 22,48 & 0,86 & 69,20 & 5,79 & 75,24 & 1,11 \\
\hline & habitación & 26,06 & 2,01 & 23,42 & 0,79 & 67,67 & 4,91 & 73,19 & 1,51 \\
\hline & terraza & 23,59 & 2,26 & 20,43 & 0,85 & 75,29 & 7,69 & 83,38 & 1,57 \\
\hline
\end{tabular}

\section{RESULTADOS}

En la Tabla 3 se presenta la evolución de los promedios de las temperaturas y humedades diarias registradas en cada una de las viviendas.

En la Figura 5 se presenta la evolución de la humedad y la temperatura de las viviendas a lo largo de las horas del día. Para lo cual se ha calculado la media de todas las mediciones tomadas a lo largo de una hora durante todos los días que duró la medición en cada vivienda.
La Figura 6 muestra las temperaturas y humedades máximas, mínimas y medias en las diferentes estancias a lo largo de los días que se realizó la toma de datos. Para lo cual se ha calculado para cada día los valores máximos, mínimos y medios.

En la Figura 7 se presenta el porcentaje de confort de la vivienda durante las horas de día y de noche en las tres estancias que han sido medidas. Como ya se explicó en metodología se ha empleado la ISO 7730 para el análisis de los datos.
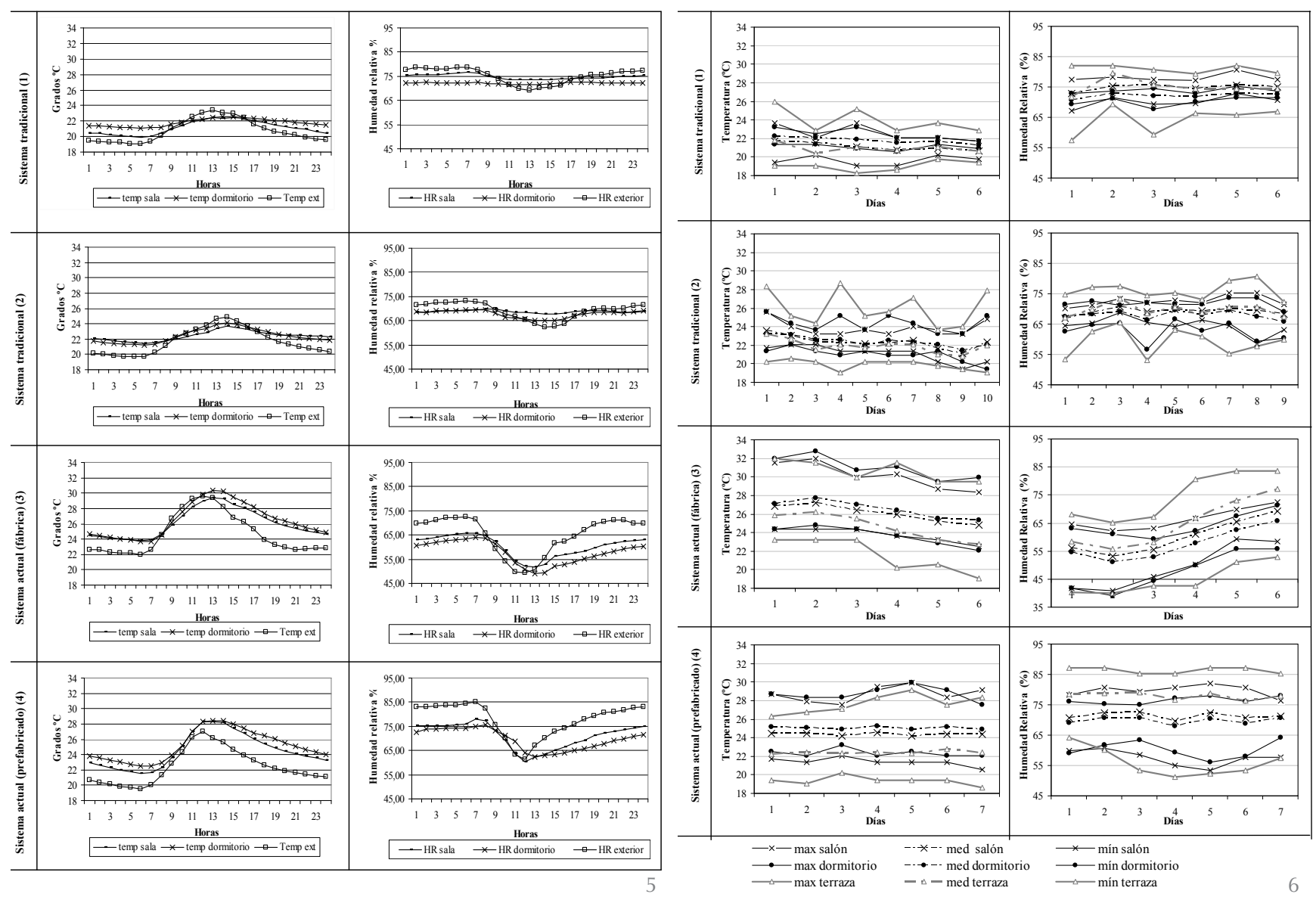

5. Evolución de la humedad y temperatura media de las cuatro viviendas a lo largo de las horas del día (julio de 2004) Santa Ana Costa Rica.

6. Valores medios, máximos y mínimos de la temperatura y humedad durante los días que se realizaron las mediciones en las diferentes viviendas. 
7. Porcentaje de confort obtenido según la ISO 7730 de la vivienda durante las horas de día y de noche.

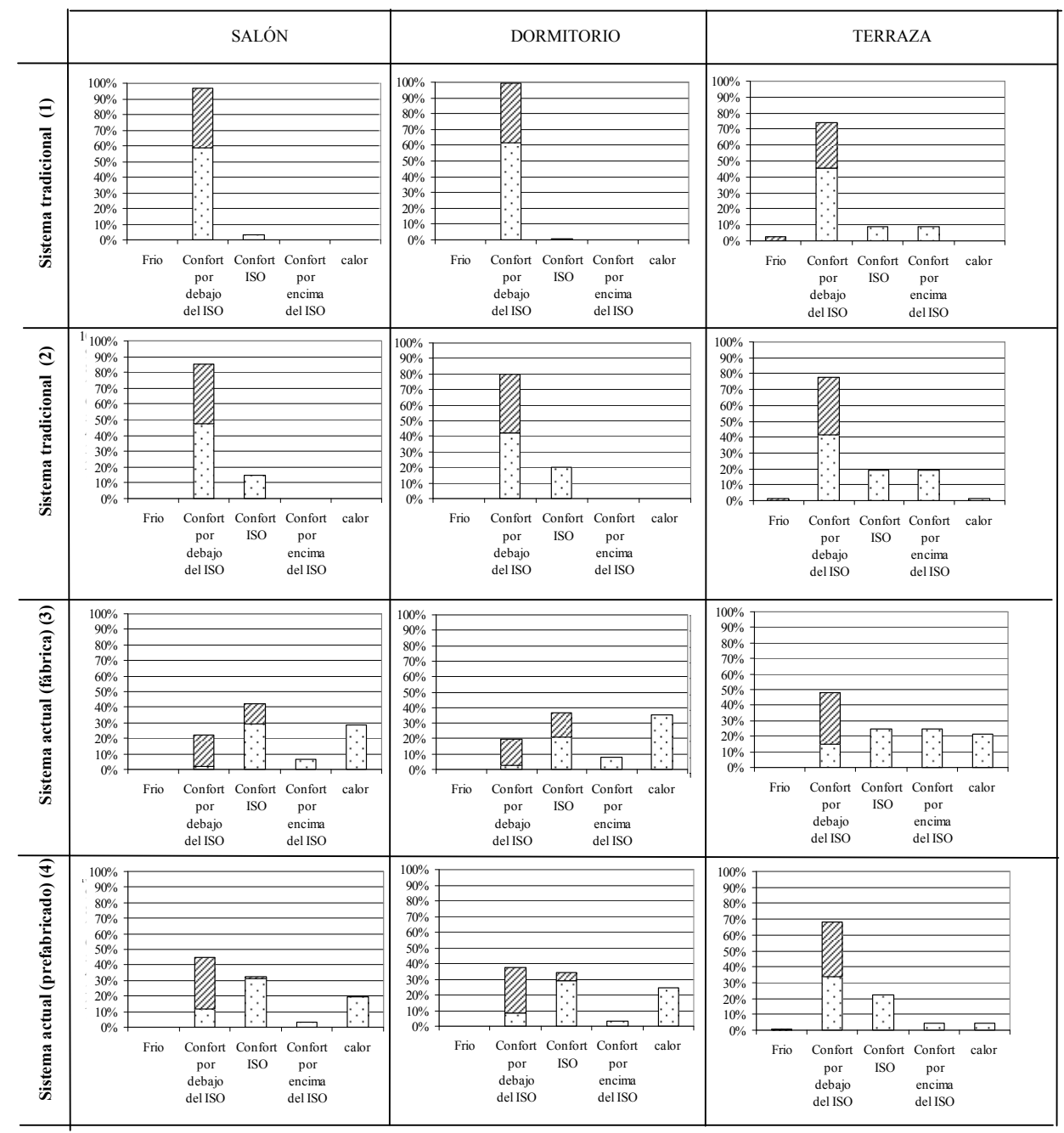

\section{\begin{tabular}{ll|}
7 & 国Nocturno \\
\hline Diurno
\end{tabular}}

\section{DISCUSIÓN}

Analizando los datos climáticos de la zona (Tabla 1) y las Figuras 6, 7 y 8 se deduce que en Santa Ana-Ciudad Colón las viviendas deberían tener muros pesados y cubiertas aisladas que protejan al usuario de las altas temperaturas en las horas centrales del día. Además el confort puede mejorar con un diseño adecuado que asegure las estrategias de ventilación cruzada que pueden disminuir las altas temperaturas o la sensación de calor en los momentos más desfavorables. Estos resultados apoyan los obtenidos por Stagno y Ugarte (12) para las zonas tropicales (especialmente en Costa Rica) en donde concluyen que las estrategias constructivas y de diseño debe centrarse en obtener viviendas que aseguren un resguardo frente a la lluvia y que minimicen el calor mediante estrategias de ventilación cruzada y con sistemas constructivos y materiales adaptados al clima.

Si se analiza las recomendaciones de Mahoney (8) se observa que: 1) solamente las viviendas con sistemas constructivos tradicionales están orientadas Norte-Sur, 2) Las aberturas de la vivienda 3 (sistemas constructivos actuales) son superiores al $40 \%$ y tanto estas como las de la vivienda 4 están orientadas, una gran parte de ellas al oeste. 3) Las aberturas en las viviendas 3 y 4 y en la ampliación de la 2 vivienda no están protegidas contra la luz directa y la lluvia mediante corredores o aleros grandes.

Si se analiza la amortiguación de las temperaturas más altas, se observa un comportamiento diferente en los tres sistemas analizados. En las viviendas de muros de adobe (Figura 5) la temperatura interior es muy constante a lo largo del día, amortiguando las temperaturas mínimas de primeras y últimas horas y las máximas de las horas centrales. De esta manera el rango de temperatura entre la media de los valores máximos y mínimos es inferior a $2{ }^{\circ} \mathrm{C}$ (Tabla 3). Esta amortiguación, en cambio no es efectiva en las viviendas con sistemas constructivos actuales. En estos dos casos (Figura 5) la temperatura exterior es menor que la interior en las ho- 
ras centrales del día. El tipo de cubierta y de muros (que facilitan esta captación) unido a la falta de ventilación efectiva de la vivienda son factores fundamentales en el hecho de que la vivienda capte el calor en las horas centrales y no sea capaz de disiparlo.

En el análisis del confort térmico en el interior de la vivienda (Figura 7) se observa un comportamiento diferente en las viviendas con un sistema constructivo tradicional con respecto a los sistemas actuales. Las cuatro viviendas analizadas presentaron un comportamiento térmico no satisfactorio como muestra que más del $50 \%$ de los datos se encuentran fuera del intervalo de confort considerado por la ISO 7730 (3). La respuesta del sistema constructivo es diferente:

1. En el interior de las dos viviendas con sistema tradicional se observa que en el $100 \%$ del tiempo de estudio las viviendas se encontraron en de los rangos establecidos por la norma ISO 7730 (3) y del ASHRAE Std 55 (11) como confort o sensación térmica confortable. No obstante, la inercia de este sistema constructivo colabora en amortiguar las temperaturas más bajas de la noche pero de la misma manera amortigua las más altas del día. De esta manera, en algunos momentos del día, el confort en el exterior de la vivienda es mayor que en el interior, en donde la sensación térmica es más fresca.

2. La estrategia climática pasiva de las viviendas de sistemas constructivos actuales es inversa. Estas viviendas no son capaces de atenuar el rigor climático durante las horas centrales del día. De hecho, la falta de confort por calor en el exterior $(23 \%$ en la vivienda 3 y $5 \%$ en la vivienda 4) es menor que en el interior $(29 \%$ en el salón de la vivienda 3 y $20 \%$ en el interior de la vivienda 4) (Figura 7). En ambos casos es en el dormitorio donde el confort es menor. Estos resultados pueden ser debidos a varias causas, algunas de las cuales ya han sido analizadas:

a) El movimiento del aire en el interior de la vivienda puede estar minimizado o impedido. Como ya se ha comentado la ventilación es una estrategia pasiva indispensable para aumentar el confort térmico y humídico dentro de la edificación, y, aunque en las viviendas 3 y 4 presentan aperturas lumínicas suficientes, no todas las ventanas son practicables. De hecho, en la vivienda 3 la ventana que se encontraba en la cubierta no podía ser abierta y únicamente era empleada como tragaluz. Además, esta casa permanece cerrada durante las horas centrales del día. En la vivienda 4 la ventilación cruzada, como se ha remarcado anteriormente, fue sacrificada por la ampliación de la vivienda.

b) La cubierta es ligera pero no está bien aislada. Para obtener un mejor comportamiento de la cubierta se puede actuar de tres formas diferentes (4): a) minimizar la absorción solar del material variando el material o el color; b) aumentar la ventilación en la cámara de aire entre las dos capas de cubierta (en este caso chapa metálica y falso techo); y/o c) aumentar la cámara de aire o el aislamiento del elemento de cubierta. En un estudio previo de simulación realizado con los datos de la zona se concluyó que el pintar la chapa metálica de colores claros influía en una disminución de las máximas temperaturas en el interior de la vivienda en las horas centrales debido a una disminución de la absorción solar (13). Esta disminución puede llegar a ser de hasta $3^{\circ} \mathrm{C}$ menos (4). Para minimizar la absorción solar también es importante evitar que los faldones de la cubierta estén orientados de forma que capten una mayor radiación solar, especialmente durante la estación seca.

c) Las orientaciones de las aperturas no son adecuadas. Además no se han diseñado para asegurar las sombras en las ventanas sobre todo en aquellas donde el asoleamiento es mayor en las horas centrales del día.

Es importante incidir en que la toma de datos se ha realizado únicamente en la estación lluviosa en donde la incidencia de los rayos solares sobre la superficie es de menor intensidad, por lo que los máximos de temperatura son menores. Por lo tanto, se considera que en la época seca los resultados obtenidos para las viviendas tradicionales pueden llegar a ser más favorables, en cambio, previsiblemente las viviendas con sistemas constructivos actuales presentarán un mayor porcentaje de tiempo en el que el calor en el interior de la vivienda no sea confortable según los parámetros de la norma UNE-EN ISO 7730 (3) y la 55 de ASHRAE (11). Igualmente la tasa metabólica considerada en el estudio es 1,2 met, la relativa a una actividad sedentaria. Actividades realizadas frecuentemente en una vivienda, como las domésticas presentan una tasa metabólica superior (2,0 met). En este caso, los intervalos de confort presentarán temperaturas máximas menores que en el que se ha estudiado, por lo que los resultados obtenidos en las viviendas tradicionales serían más favorables y los obtenidos en las viviendas actuales presentarían un mayor porcentaje de tiempo fuera del confort. 


\section{CONCLUSIONES}

Los resultados obtenidos en este estudio permiten concluir que el confort térmico de las viviendas en la zona estudiada aumenta cuando éstas presentan muros exteriores pesados que aseguren una inercia térmica del edificio y cubiertas aisladas $y / o$ ventiladas que eviten la captación excesiva de calor a través de las mismas. En las viviendas analizadas, los sistemas constructivos tradicionales, basados en muros de adobe y cubierta cerámica cumplen en mayor medida estos requisitos que los sistemas constructivos contemporáneos (de bloques o prefabricados de hormigón con chapa metálica de cubierta). Estos diseños modernos parecen menos sensibles a parámetros bioclimáticos al priorizar variables económicas, normativas y de diseño.

El sistema actualmente más extendido presenta confort térmico deficitario, presentando en la mayoría del tiempo falta de confort por calor en las estancias interiores. La cubierta parece ser el factor clave en el confort. Se considera que los materiales empleados, el color, la ventilación de la misma y la falta de aislamiento son las principales características de las cubiertas a este respecto. No obstante también tiene importancia la sombra que dicha cubierta puede arrojar sobre las aperturas verticales.

Se recomienda que las líneas de investigación futuras se orienten a: 1) determinar cómo puede variar el confort térmico con una vivienda de bloque de hormigón o prefabricada con cubierta de hierro zincado cambiando las variables de ventilación, color de la lámina y el aislante. 2) analizar la viabilidad de recuperar construcciones con valores tradicionales y bioclimáticos, tanto en el diseño como en la selección de materiales aplicando la normativa actual de edificación.

\section{AGRADECIMIENTOS}

Esta investigación se ha realizado gracias a los fondos del proyecto $\mathrm{N}^{\circ} 330-\mathrm{A} 4-076$ (2004-2005) subvencionado por la Vicerrectoría de Investigación de la Universidad de Costa Rica (UCR). En el momento de realizar el proyecto todos los autores estábamos vinculados a esta institución.

\section{BIBLIOGRAFÍA}

(1) McMullan, R.: Environmental Science in Building. Ed. Hart McLeod. Cambridge, Reino Unido, 376 pp., 2002.

(2) Fanger, P. O.: Thermal Comfort, McGraw-Hill Co.; New York, 1973.

(3) UNE-EN ISO 7730: 2006. Ergonomía del ambiente térmico: Determinación analítica e interpretación del bienestar térmico mediante el cálculo de los índices PMV y PPD y criterios de bienestar térmico local.

(4) Givoni, B.: Man, Climate \& Architecture. Ed Van Nostrand Reinhold Company. Nueva York, Estados Unidos, 483 pp., 1981.

(5) Olgyay, A.; Olgyay, V.: Solar Control and Shading Devices. Princenton University press. New Jersey, Estados Unidos, 201 pp., 1957.

(6) Rodriguez Viqueira, M.; Figueroa Castrejón, A.; Fuentes Freixanet, V.; Castorena Espinosa, G.; Huerta Velázquez, V.; García Chávez, J. R.; Rodríguez Manzo F.; Guerrero Baca, L. F.: Introducción a la Arquitectura Bioclimática. Ed. Limusa Noriega Editores. Mexico D.F. México, 204 pp., 2001.

(7) Germer, J.: Estrategias pasivas para Costa Rica: Una aplicación regional del diseño bioclimático. Imprenta Carcemo, Costa Rica, 89 pp., 1986.

(8) Koenigsberger, O. H.; Ingersoll, T. G.; Mayhew, A.; Szokolay, S.V.: Viviendas y edificios en zonas cálidas tropicales. Ed. Paraninfo, Madrid, España, 329 pp., 1977.

(9) Olgyay, V.: Desing and Climate. Princenton University press. New Jersey, Estados Unidos, 250 pp., 1963.

(10) Fournier, L.: Datos de humedad relativa y temperatura máxima y mínima media mensual en Ciudad Colón. Registro particular tomados desde 1957 a 2002. Luis Fournier, Catedrático de Facultad de Física de la Universidad de Costa Rica.

(11) ANSI ASHRAE (The American Society of Heating, Refrigerating and Air-Conditioning Engineers) Standard 55: 2004. Thermal Environmental Conditions for Human Occupancy.

(12) Stagno, B.; Ugarte, J. (2003): Arquitectura rural en el trópico, enclaves bananeros de Costa Rica. Ed: Instituto de Arquitectura Tropical, $1^{\text {a }}$ ed. San José, Costa Rica, 140 pp.

(13) Rodrigo, B. G.; Sanabria, J. C.; Umaña, M.: Informe Final del proyecto Biomonitoreo de diferentes sistemas constructivos en San José. Proyecto 330-A4-076 de la vicerrectoría de investigación de la Universidad de Costa Rica. San José, Costa Rica, 60 pp., 2005. 